\title{
«La ballena vuelve al museo»; proceso de diseño y cálculo de una estructura ligera
}

\author{
"The whale comes back to the museum"; design and calculation process \\ of a lightweight structure
}

$\underline{\text { I. Costales Calvo }}^{(*)}$

RESUMEN

El texto describe el proceso de diseño y cálculo de una estructura metálica ligera que soporta otra estructura aún más ligera: el esqueleto de una ballena. Una pieza que ha sido conservada durante más de 150 años. Se encuentra suspendida de otra estructura metálica bidireccional y singular, que resuelve grandes luces: el techo del edificio Fòrum 2004 de Barcelona. Para encontrar la solución más adecuada se ha utilizado un programa de cálculo no lineal. Gracias a un diseño eficiente y a un exhaustivo análisis se ha conseguido que la estructura principal trabaje a tracción, eliminando la posibilidad de pandeo, reduciendo así la sección resultante a la necesaria para trabajar a flexo-tracción, por lo que la estructura ha podido quedar oculta dentro del esqueleto. Durante la fase de diseño se hicieron propuestas adecuadas para poder transportar las piezas y soldarlas o ensamblarlas fácilmente. Finalmente se describe la puesta en obra de la estructura, que se realizó en un fin de semana, para poder sostener a uno de los iconos más queridos por los niños de Barcelona: «La balena Brava».

Palabras clave: esqueleto; ballena; diseño estructural; estructura tensada; sistema no lineal; estructura ligera; puesta en obra.

\section{ABSTRACT}

The text describes the design process and calculation of a light metal structure that supports an even lighter one: the skeleton of a whale, a specimen that has been preserved for more than 150 years. Both of them are suspended from another bidirectional and unique metallic structure that solves large spans: the roof of the Forum 2004 building in Barcelona. To find the most suitable solution, a non-linear calculation program was used. Moreover it has been achieved that the main structure to work in tension, removing the possibility of buckling, thereby reducing the resulting section needed to work in Flexural strength, so the structure could be hidden inside the skeleton. During the design phase several proposals were considered to make it easier to transport the pieces as well as to weld or join them. The text also describes the site works, which were completed in just a weekend in order to support one of the most beloved icons of Barcelona: "La balena Brava”.

Keywords: skeleton; whale; structural design; stay structure; nonlinear system; light weight structure; project management.

(*) Doctor Arquitecto. Profesor asociado ETSAV. Laboratori d'Innovació i Tecnologia a l'Arquitectura - Universitat Politècnica de Catalunya (UPC-BarcelonaTech), Barcelona (España).

Persona de contacto/Corresponding author: nacho.costales@upc.edu (I. Costales Calvo)

ORCID: http://orcid.org/oooo-0oo1-5746-2351 (I. Costales Calvo)

Cómo citar este artículo/Citation: Costales Calvo, I. (2016). «La ballena vuelve al museo»; proceso de diseño y cálculo de una estructura ligera. Informes de la Construcción, 69(546): e189, doi: http://dx.doi.org/10.3989/ic.16.050.

Copyright: (C) 2017 CSIC. Licencia / License: Salvo indicación contraria, todos los contenidos de la edición electrónica de Informes de la Construcción se distribuyen bajo una licencia de uso y distribución Creative Commons Attribution License (CC BY) Spain 3.o. 


\section{INTRODUCCIÓN}

Entre el año 2009 y el 2010 el Museu de Ciències Naturals de Barcelona inició un proceso de modernización, incorporando una nueva sede en el edificio Fòrum de les Cultures, obra de los arquitectos Herzog \& de Meuron (1). Es esta misma prestigiosa firma suiza quien se responsabiliza del diseño de la forma y posición del esqueleto de la ballena (2) en el nuevo museo. En junio del 2010 se cerró temporalmente el Museu del Parc de la Ciutadella para, entre otros trabajos, trasladar la ballena a su nueva ubicación. Una vez desmontada cada pieza se le aplicó un tratamiento de restauración a los huesos (3) (4). Así entre el 4 y el 10 de julio del 2011 el Fòrum cerró sus puertas y en el acceso se colocó un letrero que explicaba al público: «La balena torna al Museu».

La estructura portante del esqueleto de la ballena del Museu de Ciències Naturals de Barcelona es un encargo poco frecuente en la carrera profesional de consultoría estructural. Pocas veces es necesario definir una estructura ligera que se suspende de otra más pesada para soportar una estructura natural: el esqueleto de un animal. El objetivo del proceso de diseño adoptado es buscar la solución más adecuada que dé respuesta a los esfuerzos que la posición del esqueleto genera en el espacio, usando los puntos fuertes de la estructura existente, y elementos sensibles con el encargo. El artículo se ha escrito con el claro propósito de mostrar al lector este proceso, desde su diseño hasta la puesta en obra, y de cómo se ha conseguido dar respuesta a los problemas que se presentaron. La lectura de este documento es también recomendable para consultores nobeles, pues para alcanzar una correcta metodología de análisis es necesario tener oficio y experiencia (5). En algunos de los capítulos se hace referencia a actitudes que adopta el consultor estructural en cada fase del análisis.

Para dimensionar la solución final se describe el proceso de recogida de datos, de predimensionado manual y del uso de un software de cálculo matricial no lineal de barras, que ha garantizado los aspectos resistentes y constructivos del conjunto. El resultado es por sí mismo una solución única que da respuesta a la forma, y que integra Arquitectura y Estructura.

\section{METODOLOGÍA EMPLEADA}

\subsection{Datos técnicos}

La ballena, un rorcual común (MZB 83-3084 Balaenoptera physalus) varó en la playa de Llançà hace ahora unos 155 años, concretamente el 11 de junio de 1862 (6). El esqueleto de la ballena, que entonces decidió conservarse en diferentes enclaves, tiene una longitud de aproximadamente 18,3 m: 4,3 $\mathrm{m}$ la cabeza, 4,0 m el tórax y 10,0 $\mathrm{m}$ la cola ${ }^{1}$. Pesa en total unos $11,60 \mathrm{kN}$. El reparto del peso no es lineal: la parte más pesada es la cabeza $(4,83 \mathrm{kN})$, después el tórax con las «manos $»^{2}(3,67 \mathrm{kN})$ y por último la cola (unos $3,10 \mathrm{kN}$ ). Estos datos se recogen en la Tabla 1:

Tabla 1. Características del esqueleto desglosadas por partes.

\begin{tabular}{|l|c|c|c|}
\hline & $\begin{array}{c}\text { Longitudes } \\
(\mathbf{m m})\end{array}$ & Peso $(\mathbf{k N})$ & $\mathbf{q}(\mathbf{k N} / \mathbf{m})$ \\
\hline Cabeza & 4.000 & 4,83 & 1,21 \\
\hline Tórax & 4.300 & 3,67 & 0,85 \\
\hline Cola & 10.000 & 3,10 & 0,31 \\
\hline Total & 18.300 & 11,60 & \\
\hline
\end{tabular}

El vertebrado se encontraba colgado del techo de la sala del Museu del Parc de la Ciutadella en una posición completamente recta. Esta posición es anti natural, pero es sin duda la más usual en la mayoría de los museos del mundo, pues es la que menos altura libre de sala requiere. Además el esqueleto se había expuesto sin dejar un espacio entre sus vértebras, tal como se puede apreciar en la Figura 1.

Las ballenas, al igual que el resto de los vertebrados, tienen unos discos intervertebrales que son los responsables de que la columna vertebral sea flexible, puesto que representan un amortiguamiento cartilaginoso de la misma (7). El material que lo forma es blando y fácilmente degradable, por lo que no se puede conservar. Estos discos tienen un grosor de aproximadamente $20 \mathrm{~mm}$. Si se considera que el cetáceo tiene 53 vértebras ${ }^{3}$, fácilmente quedará demostrado que la columna vertebral era, en el Museu del Parc de la Ciutadella, aproximadamente un metro más corta que cuando el cetáceo varó.

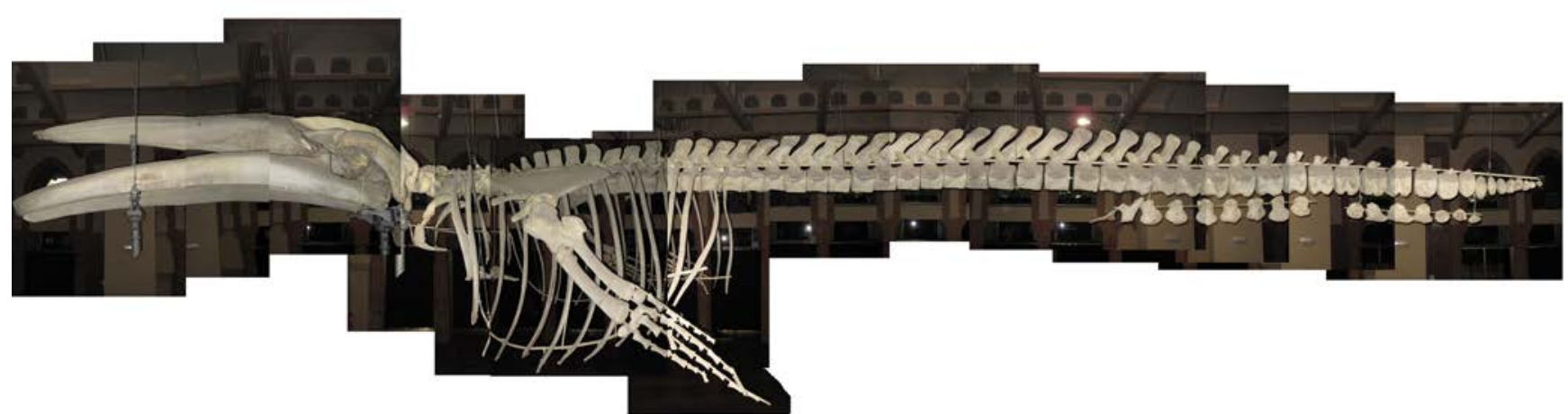

Figura 1. Fotomontaje del estado inicial de la ballena en el Museu del Parc de la Ciutadella. En la imagen se puede apreciar la ausencia de la mayoría de los discos intervertebrales. Autor: GEA.

\footnotetext{
${ }^{1}$ Comunicación personal, confirmados durante el proceso de montaje de la exposición.

2 Más comúnmente denominadas «Aletas».

3 Extraído de los paneles del Museu Blau referentes al esqueleto de la ballena.
} 
La cabeza es sin duda la parte más delicada del esqueleto. Son huesos pesados y largos que se pueden desestabilizar fácilmente, puesto que el centro de gravedad del conjunto queda desplazado del centro geométrico. El vómer, que es el hueso central superior por encima de las dos mandíbulas, había sido dañado en manipulaciones anteriores y, a pesar de que ahora había sido muy bien restaurado, suponía un punto débil para el futuro (8) (9).

\subsection{Buscando la forma}

El esqueleto ocupa una posición privilegiada dentro del museo, tal como se muestra en la Figura 2, da la bienvenida al público en la doble altura de las escaleras del acceso principal. Por decirlo coloquialmente, se convierte en el «gancho» de la exposición ${ }^{4}$. En el Museu Blau el esqueleto se dispone de una manera curva, que es más natural para un cetáceo en libertad. La cabeza queda situada en el techo de la planta del acceso mientras que la cola queda en el techo del nivel superior, aparentando que el animal se sumerge buscando el mar, que se encuentra muy próximo al acceso principal del museo, hacia donde mira la cabeza (10). Así, todo el eje de la columna vertebral del esqueleto, que al ser curvo forma un plano, que se propone inclinado, por lo que el centro de gravedad del conjunto queda fuera del mismo (11) (12).

La posición final resulta muy atractiva porque los visitantes, cuando entran por primera vez por la puerta del Museu, se encuentran al vertebrado dirigiéndose hacia ellos. Mientras suben por las escaleras que les llevan a la venta de entradas pueden recorrer el conjunto de cabeza a cola como si estuviesen dentro del vientre del animal para, finalmente, una vez alcanzan el primer piso, poder recorrer visualmente de nuevo el esqueleto a la altura de su espalda, esta vez desde la cola a la cabeza.

La posición estaba definida en el espacio por el despacho de arquitectos Herzog \& de Meuron. Era necesario definir una estructura horizontal que consiguiese que los huesos quedasen fijos en su posición, así como un sistema de tirantes para soportarla desde el techo (13). La estructura horizontal está compuesta por el «Arco» y por la «Silla». El «Arco» es un tubo que se sitúa en el eje del esqueleto del animal y que debe de ser previamente curvado. Se dispone dentro de los huesos, por lo que casi la totalidad de las vértebras deben ser perforadas. El tamaño del tubo debía ser menor a $50 \mathrm{~mm}$. El límite lo imponía el tamaño de las vértebras y el espacio restante mínimo necesario cercano al taladro para que las piezas no quedasen dañadas (14) (15). Se optó por elegir un diámetro de $44 \mathrm{~mm}$ con un espesor de $2,7 \mathrm{~mm}$, puesto que era el canto más grande que se encontraba en stock.

Por lo que se refiere a la «Silla», que es el sistema de soporte de la cabeza, se propuso la subestructura que se muestra en la Figura 3. Los dos tubos centrales, casi verticales, que tienen en su extremo superior una chapa doblada en forma de «V» de $1 \mathrm{~mm}$ de grosor, sirven para dar soporte al vómer. Los tubos curvados perimetrales sirven para dar soporte continuo casi a la totalidad de las dos mandíbulas. Los dos sistemas de la derecha en forma de «V» invertida sirven para soportar la parte posterior del mentón, perforándolo parcialmente, consiguiendo que el esqueleto no deslice, puesto que la «Silla» está inclinada en el espacio hacia adelante.

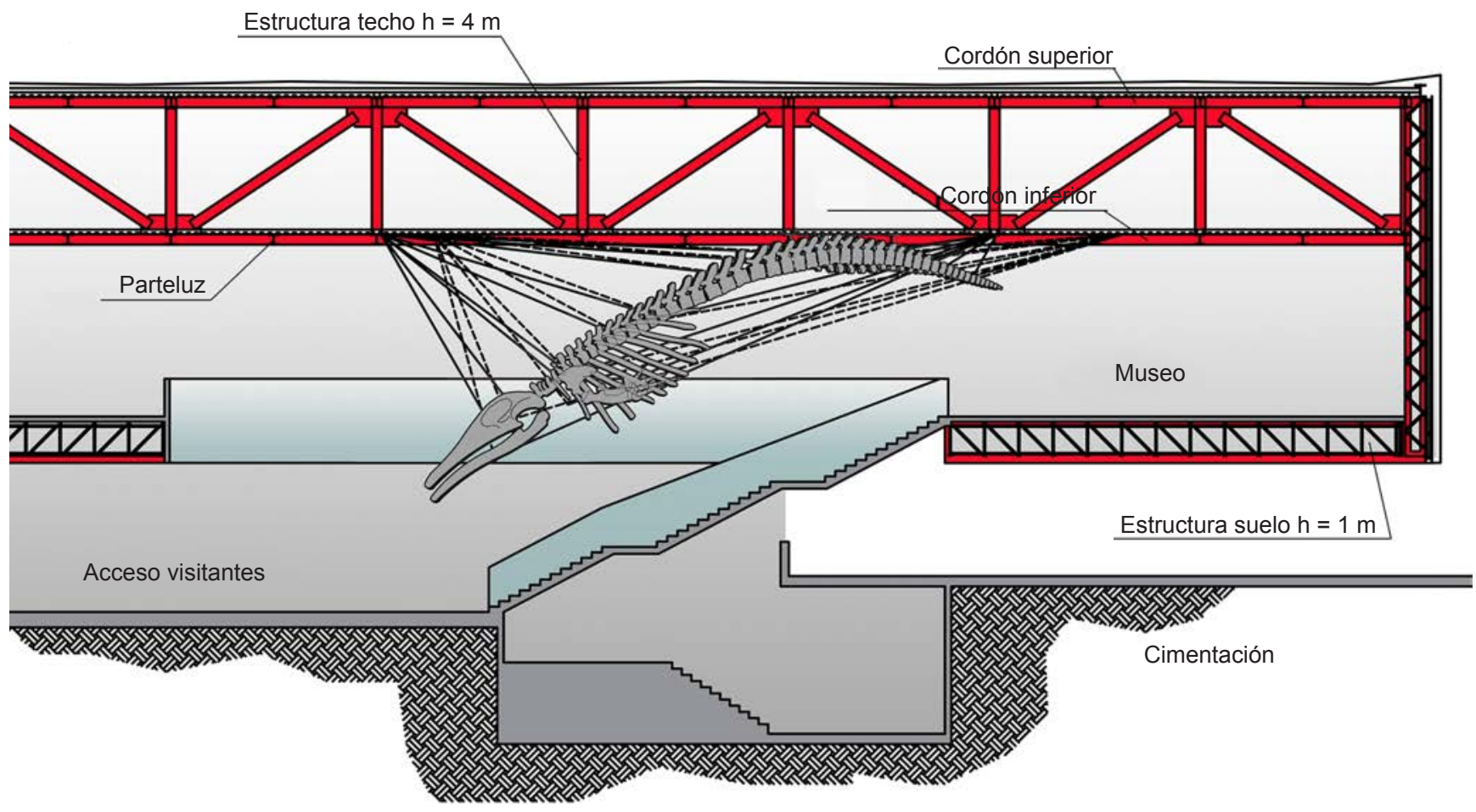

Figura 2. Sección transversal del edificio donde se puede observar la situación de la ballena.

\footnotetext{
${ }^{4}$ Uno de los comentarios más repetidos por los niños, buscando la protección de los padres, cuando entran por primera vez al museo, es: «¿̉No se caerá?» La respuesta de los padres tranquiliza a los niños. Algunos padres les dicen a sus hijos el peso del conjunto, pues se encuentra anotado en la pared.
} 


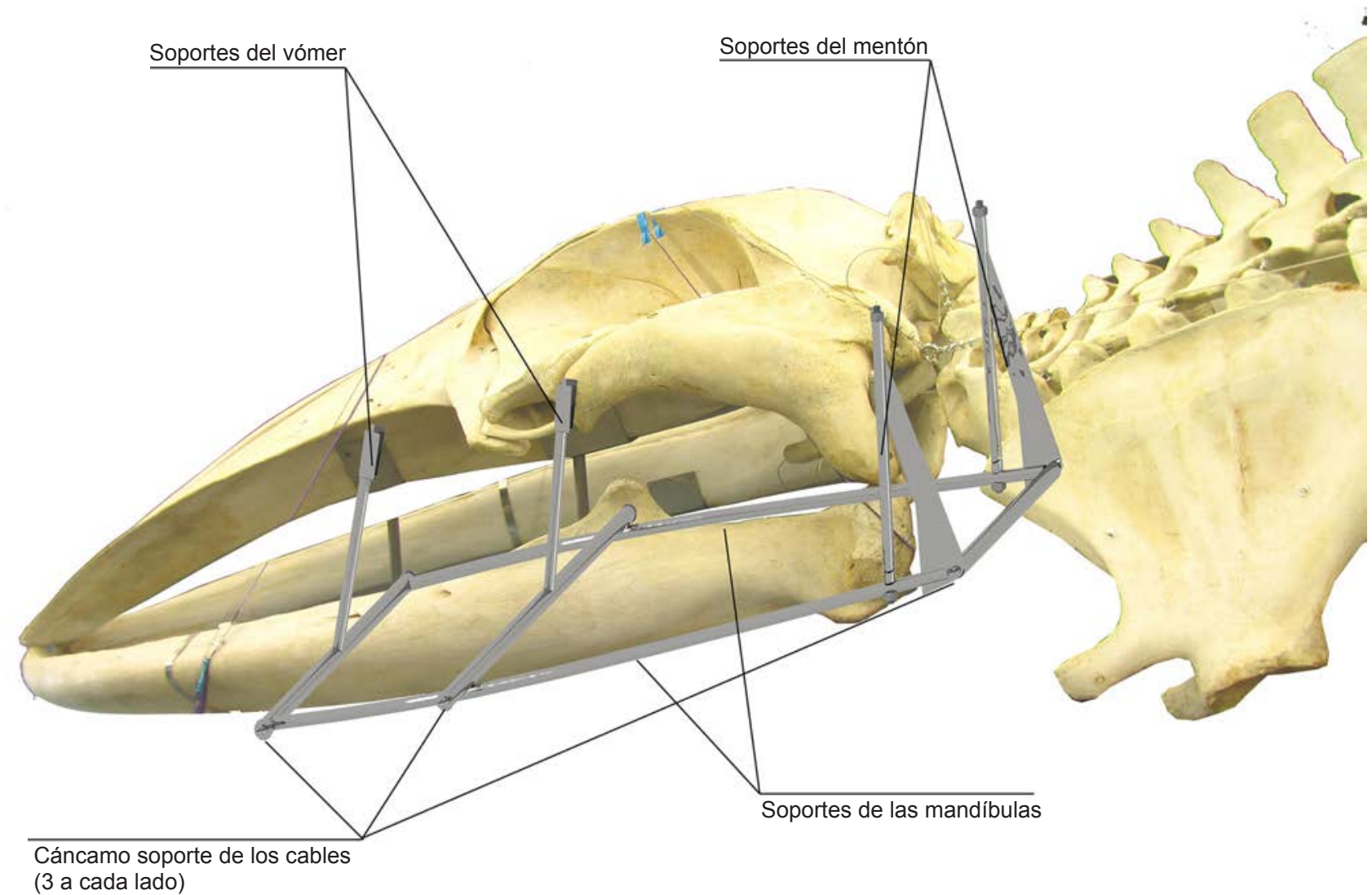

Figura 3. Sistema de soporte de la cabeza o «Silla» superpuesto con el cráneo.

La estructura de soporte de la ballena debía ser ligera (16). Para poder definir el sistema de tirantes más eficaz fue necesario antes estudiar la composición estructural del techo, que es el soporte del soporte de la ballena. Está compuesto por una estructura de $4.000 \mathrm{~mm}$ de canto, formado por vigas metálicas en celosía en dos direcciones ortogonales entre sí, que tienen montantes compartidos cada $6.000 \mathrm{~mm}$ tal como puede apreciarse en la Figura 4 (17). En el cordón superior hay un forjado colaborante que forma el techo del edificio, $\mathrm{y}$ en el cordón inferior hay otro forjado igual que hace de suelo, que sirve de soporte a las instalaciones del edificio: es el nivel desde el que se colgará la ballena. Como los espacios entre las vigas que resultan tienen dimensiones de $6 \times 6 \mathrm{~m}^{2}$, existe un parteluz central en cada piso, biarticulado, y siempre en la misma dirección, consiguiendo luces para el forjado unidireccional de hormigón de $3.000 \mathrm{~mm}$, más acordes para las cargas de cada piso.

El eje en planta del esqueleto de la ballena no sigue los ejes principales de la estructura del techo, tal como se explicará más adelante. Así pues existían tres posibles opciones de apoyo: apoyo directo sobre el forjado colaborante, apoyo en el tramo central de los cordones y apoyo en nudo.

a) Apoyo directo sobre el forjado colaborante (posición 1 en la Figura 4). Esta solución era comprometida: el forjado de $110 \mathrm{~mm}$ de espesor en los nervios principales soporta cargas de mediana intensidad. Si se optaba por usar tacos químicos, de reducida eficacia para este caso en concreto, puesto que son tacos trabajando a tracción y con colocación en techo, o tacos mecánicos, no quedaría espesor suficiente para garantizar los esfuerzos. Existía una variante: cons- taba de una placa inferior y de otra contra placa superior unidas mediante barras soldadas pasantes por unos taladros previamente ejecutados en el forjado, sobre las que se soldaba una orejeta vertical que servirá de gancho. Los equipos de climatización instalados que se encuentran sobre este forjado podían impedir su colocación. Y si no había interferencia, la perforación del forjado podía desembocar en un punto débil para la impermeabilización, con posibilidad de goteras si algún equipo tuviese pérdidas. Por todo lo anterior parecía lógico no usar este tipo de apoyos.

b) Apoyo en el tramo central de los cordones (posición 2 en la Figura 4), formados por perfiles HEA, HEB o HEM-30o. Como la ballena pesa $11,60 \mathrm{kN}$, si se optaba por usar cuatro apoyos, la reacción vertical de cada uno sería de unos 4,0 o 5,0 kN máximo, valor más que aceptable para una sección de $300 \mathrm{~mm}$ de calibre y de $6.000 \mathrm{~mm}$ de luz, teniendo presente que la sobrecarga de uso prevista del techo es de $2,0 \mathrm{kN} / \mathrm{m}^{2}$. Cuando se indica que la reacción vertical es de aproximadamente $5,0 \mathrm{kN}$ se tiene en cuenta que la carga en el espacio puede tener una intensidad muy superior a las verticales, porque los apoyos pueden ser cables prácticamente horizontales que no aceptan una excesiva deformación causada por su peso propio. Así pues en cada apoyo puede existir además una reacción horizontal de gran intensidad.

El cordón puede asumir reacciones horizontales en la dirección de su eje principal, puesto que se transmiten como esfuerzos axiles de tracción y de compresión, pero no puede hacerlo en la dirección perpendicular a su eje, que se traducirían en cortantes y flexiones transversales. Además existirían torsiones, puesto que el punto de aplicación se produce en la cara inferior del ala inferior del perfil en doble T. Un punto que sí puede controlar estos esfuerzos es 
el de unión entre correa y cordón (posición 4 en la Figura 4), puesto que el cruce evita las flexiones, torsiones y cortantes antes citados.

c) Apoyo en nudo (posición 3 en la Figura 4). Es sin duda la posición mejor puesto que queda completamente controlado cualquier tipo de esfuerzo. Hay que tener presente que algunos nudos tienen pasamanos inferiores para evitar la tensión transversal de las fibras de las alas inferiores. Estos pasamanos, en caso de recibir una carga vertical, podían flexionarse excesivamente, provocando el colapso del apoyo.

Una vez se definieron los tipos de apoyos más adecuados era necesario elegir un número todavía indeterminado de puntos en el techo que, además, permitiesen no desmontar las diferentes instalaciones que el edificio tiene dispuesto bajo el forjado. Los trabajos iban a ser realizados en un fin de semana y desviar las instalaciones hubiese supuesto posponer la inauguración. Tampoco era adecuado que los cables (18) quedasen al alcance de los visitantes, pues su movimiento podría resultar inadecuado para la conservación del esqueleto. Para inmovilizar al vertebrado, cuatro es el número mínimo de puntos de soporte, puesto que la pieza alcanza los $19 \mathrm{~m}$ de longitud, y al estar dispuesta de una manera no simétrica respecto al techo, es necesario estabilizar el conjunto. No era recomendable usar más puntos, puesto que en cada uno de ellos se deben de hacer trabajos de soldadura, y porque el ajuste del tesado posterior también será más ágil, tal como se puede apreciar en la Figura 5.

Tras varias reuniones de trabajo con el equipo de Arquitectura, y después de varias versiones que hicieron posible la solución final, se pudo concluir que la mejor decisión era colocar dos apoyos adelantados a la cabeza y dos por detrás de la cola, en ambos casos uno a la derecha y otro a la izquierda del eje de la columna vertebral. Si los apoyos quedaban situados entre la cabeza y la cola, en el ámbito del cuerpo, el tubo se comprimía y tendía a girar, quedando inestable el conjunto. Cuando los apoyos se alejaban del cuerpo, el tubo se traccionaba, quedando el conjunto estable. Tres apoyos se corresponden a la posición 3 y el cuarto a la posición 4 de la Figura 4, según se ha explicado en este mismo capítulo.

Como el «Arco» no queda contenido en un plano vertical sino en uno inclinado, la estructura tenderá a girar intentando recuperar la vertical del plano que lo contiene. Así que es necesario no sólo soportar cargas verticales sino también tener la posibilidad de aplicar cargas transversales, a modo de esfuerzos torsores que eviten el giro. Este problema y su forma de controlarlo se explica en la Figura 6.

Para definir los cables de soporte (19) se encontró una obra construida que podía servir como referencia: el soporte de la luminaria que hay en la entrada a Barcelona desde la autopista $\mathrm{C}_{5} 8$, por encima de la Avenida Meridiana (20). La estructura de la $\mathrm{C}_{5} 8$ utiliza dos cables principales que se unen a la cabeza de cuatro mástiles, uno en cada extremo. Desde los cables principales se lanzan otros cables que triangulan el espacio intermedio, que son los que soportan la luminaria, y que convierten los cables principales en funiculares. La apariencia de la estructura de la $\mathrm{C}_{5} 8$ es la de dos vigas inclinadas en celosía muy ligeras. La solución final que se adoptó para soportar la ballena, diferente a la anterior, fue unir cada uno de los cuatro apoyos con varios puntos del tubo, tantos como fuesen necesarios para que el tubo resultase capaz de resolver los esfuerzos, y se pudiese controlar el fenómeno torsional que se explicó anteriormente.

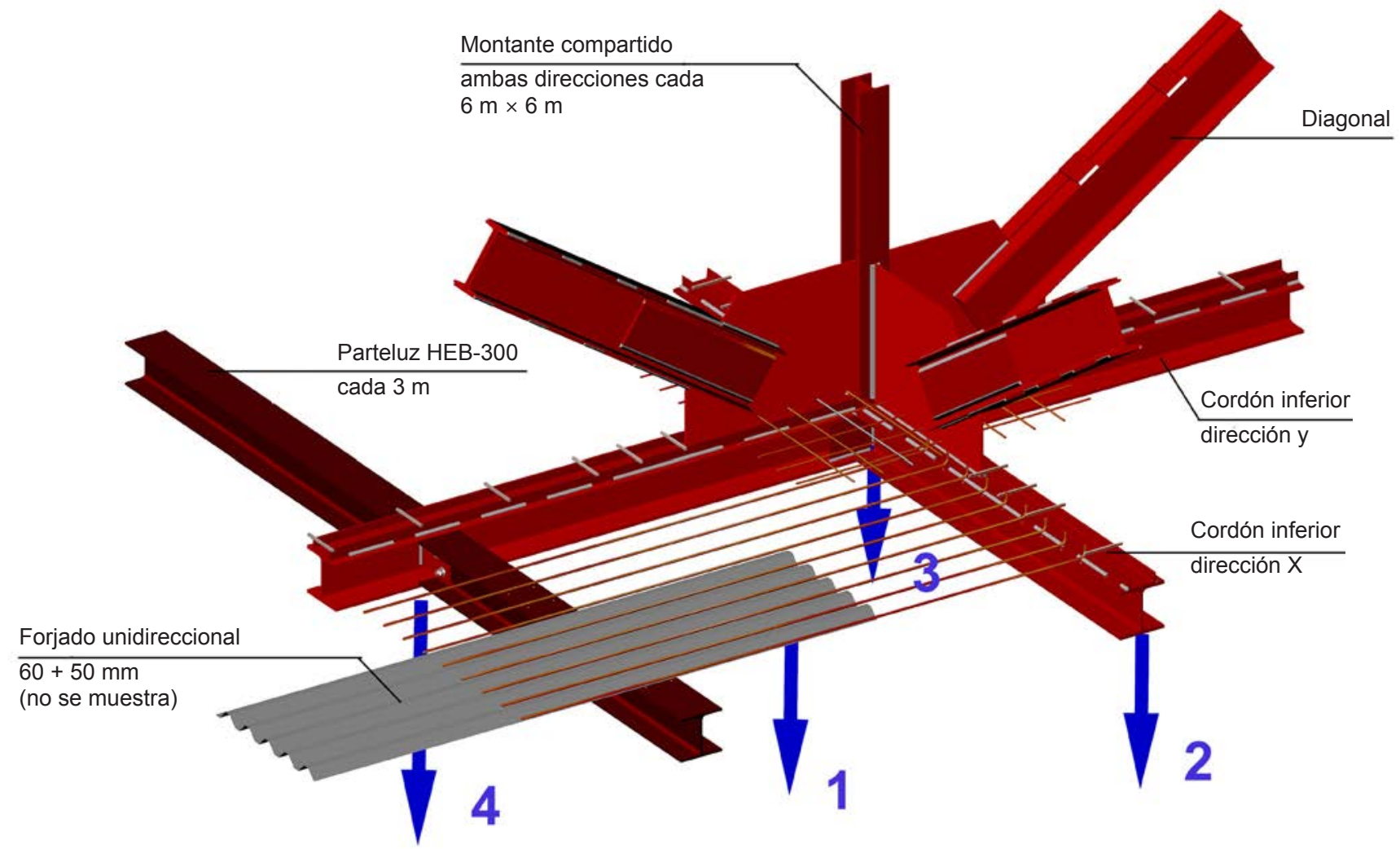

Posibles situaciones para los apoyos de los tirantes

Figura 4. Explicación de los posibles tipos de apoyos sobre la estructura principal del techo. Detalle del cordón inferior. 


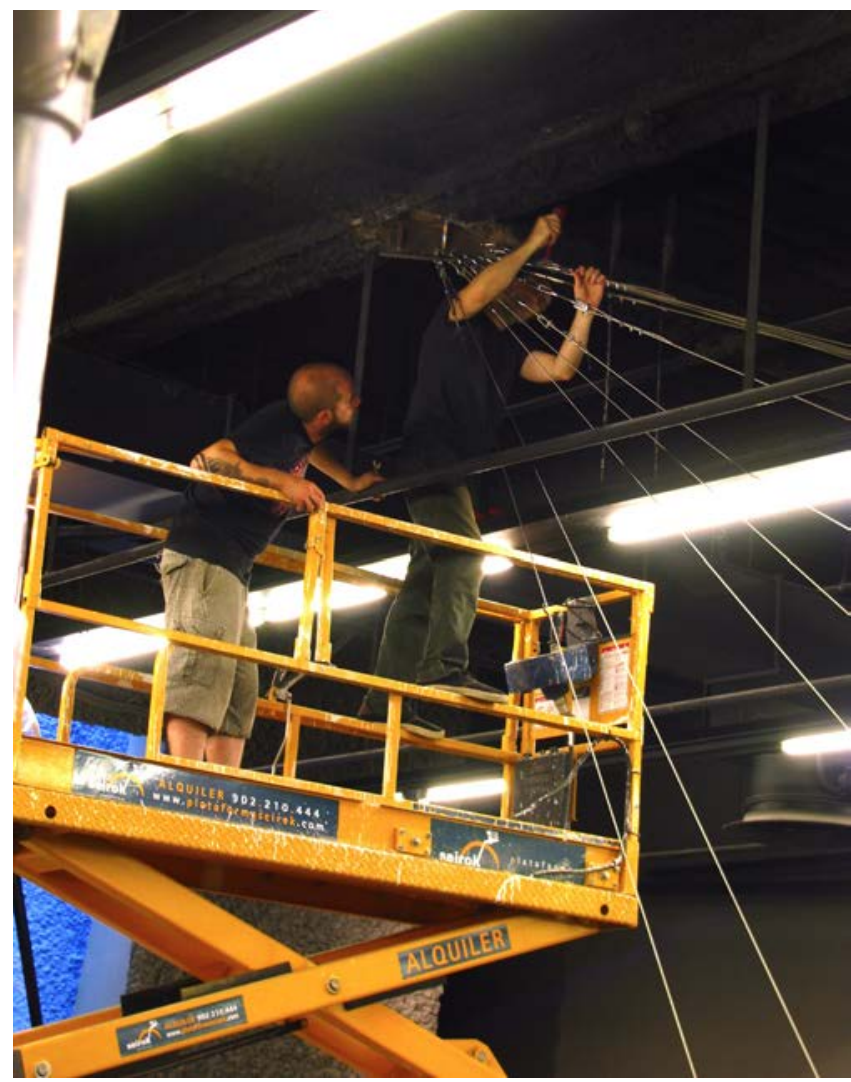

Figura 5. Proceso de ajuste de los cables en obra por los técnicos de GROP, en uno de los cuatro apoyos.

Una vez encontrada la forma era necesario, antes de proceder al cálculo definitivo, realizar un predimensionado de los elementos que componen la estructura, con objeto de aproximar la rigidez final de las barras, consiguiendo desde el inicio un reparto de esfuerzos muy cercano al de la solución final.

\subsection{Números gordos}

$\mathrm{El}$ «Arco» únicamente se podía disponer en el tórax y en una parte de la cola. Las cargas uniformemente distribuidas indicadas en la tesado 1 generarán esfuerzos de flexión y de cortante en el tubo. Como el tubo queda dispuesto en el espacio de una manera asimétrica, y como los tirantes lo pretraccionarán, es seguro que además trabajará a esfuerzo axil. Los límites de resistencia para el tubo CHS-44x2,7 (21) son los que se describen en la Tabla 2:

Tabla 2. Cálculo de los esfuerzos últimos para el tubo CHS- $44 \times 2,7$.

\begin{tabular}{|l|l|l|l|l|l|}
\hline $\mathbf{d}:$ & 44 & $\mathrm{~mm}$ & $\mathbf{A}_{\mathbf{x x}}:$ & 350 & $\mathrm{~mm}^{2}$ \\
\hline $\mathbf{t :}$ & 2,7 & $\mathrm{~mm}$ & Peso: & 0,0275 & $\mathrm{kN} / \mathrm{m}$ \\
\hline $\mathbf{f}_{\mathbf{y}}:$ & 275 & $\mathrm{~N} / \mathrm{mm}^{2}$ & $\mathbf{I}:$ & $75 \cdot 011$ & $\mathrm{~mm}$ \\
\hline $\mathbf{f}_{\mathbf{y d}}:$ & 262 & $\mathrm{~N} / \mathrm{mm}^{2}$ & $\mathbf{W}_{\mathbf{p l}}:$ & 4.612 & $\mathrm{~mm}^{3}$ \\
\hline $\boldsymbol{\varepsilon}:$ & 0,92 & & $\mathbf{N}_{\mathbf{R d}}:$ & 91,8 & $\mathrm{kN}$ \\
\hline $\mathbf{d} / \mathbf{t}:$ & 16,30 & & $\mathbf{V}_{\mathbf{p l}, \mathbf{R d}}:$ & 34,0 & $\mathrm{kN}$ \\
\hline Clase: & 1 & & $\mathbf{M}_{\mathbf{p l}, \mathbf{R d}}:$ & 1,21 & $\mathrm{kN} \cdot \mathrm{m}$ \\
\hline
\end{tabular}

El axil máximo que puede soportar el «Arco», que no puede sobrepasar nunca el límite de $91,80 \mathrm{kN}$, es función:

- De la carga distribuida que soporta: la zona del tórax está más solicitada que la zona de la cola.

- Del tipo del signo del esfuerzo: si la fuerza es de compresión el pandeo limita el esfuerzo axil máximo.

- De la longitud del tramo: a menor luz, menor tensión resultante del momento flector.

- Del límite de la deformación máxima: que queda establecido en L/300.

- De la esbeltez máxima admisible del tramo: que a compresión es 2,0 y a tracción es 3,0.

Considerando el peso del esqueleto, diez soportes y un ángulo de referencia para los tirantes igual a $25^{\circ}$, se concluye que el axil del tubo no debe de superar los 2,o kN, tal como se demuestra en la fórmula [1]. Este axil también sirvió para entender que el diámetro de los cables no debía de ser mayor de $4 \mathrm{~mm}$ (22).

$$
\mathrm{N}_{\max }=\frac{\mathrm{q}_{\mathrm{Ed}} \cdot \gamma_{\mathrm{f}} \cdot \mathrm{q}_{\mathrm{sW}}}{\mathrm{nc} \cdot \cos \alpha}=\frac{11,60 \mathrm{kN} \cdot 1,35 \cdot 1,1}{10 \cdot \cos \left(25^{\circ}\right)}=1,90 \mathrm{kN}
$$

Donde:

$\mathrm{q}_{\mathrm{Ed}}$ es el peso propio del esqueleto.

$\gamma_{f}$ es el coeficiente parcial para la acción.

$\mathrm{q}_{\mathrm{sw}}$ es el peso propio de la estructura que se estima en un $10 \%$ del peso total de la ballena ${ }^{5}$.

nc es el número de cables.

a es el ángulo aproximado de los cables.

Gracias a la Figura 7 se analizó el axil máximo que puede soportar el tubo en la zona del tórax y en la zona de la cola, y de él se obtuvo la distancia máxima necesaria entre soportes: a mayor axil máximo resistido, menor luz entre apoyos. El axil se muestra con dos ecuaciones para cada caso: $\mathrm{N}_{\mathrm{c}, \mathrm{Rd}}$ es el axil máximo a compresión, y $\mathrm{N}_{\mathrm{t}, \mathrm{Rd}}$ es el axil máximo a tracción. Para el caso de compresión las ecuaciones se solapan, porque es el fenómeno de pandeo el que limita la capacidad. En el caso de la zona de tórax el axil máximo resistente es mayor que para la zona de la cola, porque la carga debida al peso propio aplicada es mayor en el primer caso. Sin embargo, la longitud máxima admisible entre tirantes en zonas comprimidas era menor en la zona del tórax, 2,0 m, mientras que en la zona de la cola se podía llegar a 2,5 m. Para el caso de tracción las dos ecuaciones, que son independientes, descuentan la tensión producida por la flexión, y muestran el axil máximo que se puede utilizar para soportar la tracción. Si el tubo se traccionaba, en la zona del tórax se podía llegar a distancias entre cables de 2,3 $\mathrm{m}$, mientras que en la zona de la cola se podía llegar a 3,8 m con un axil menor. La conclusión más importante a la que se llegó fue que forzar la tracción del tubo, en el caso que nos ocupaba, era una mejora para el «Arco» (23), porque se reducía el número de cables necesarios, y por tanto el coste.

El tubo debía ser cortado en varias partes para facilitar el transporte y el posterior montaje, por lo que el caso de re-

\footnotetext{
${ }^{5}$ Se estableció que la estructura podía pesar un $10 \%$ del peso total de la ballena, comparando el peso de los tubos con el del esqueleto y gracias a un metraje previo de los cables.
} 


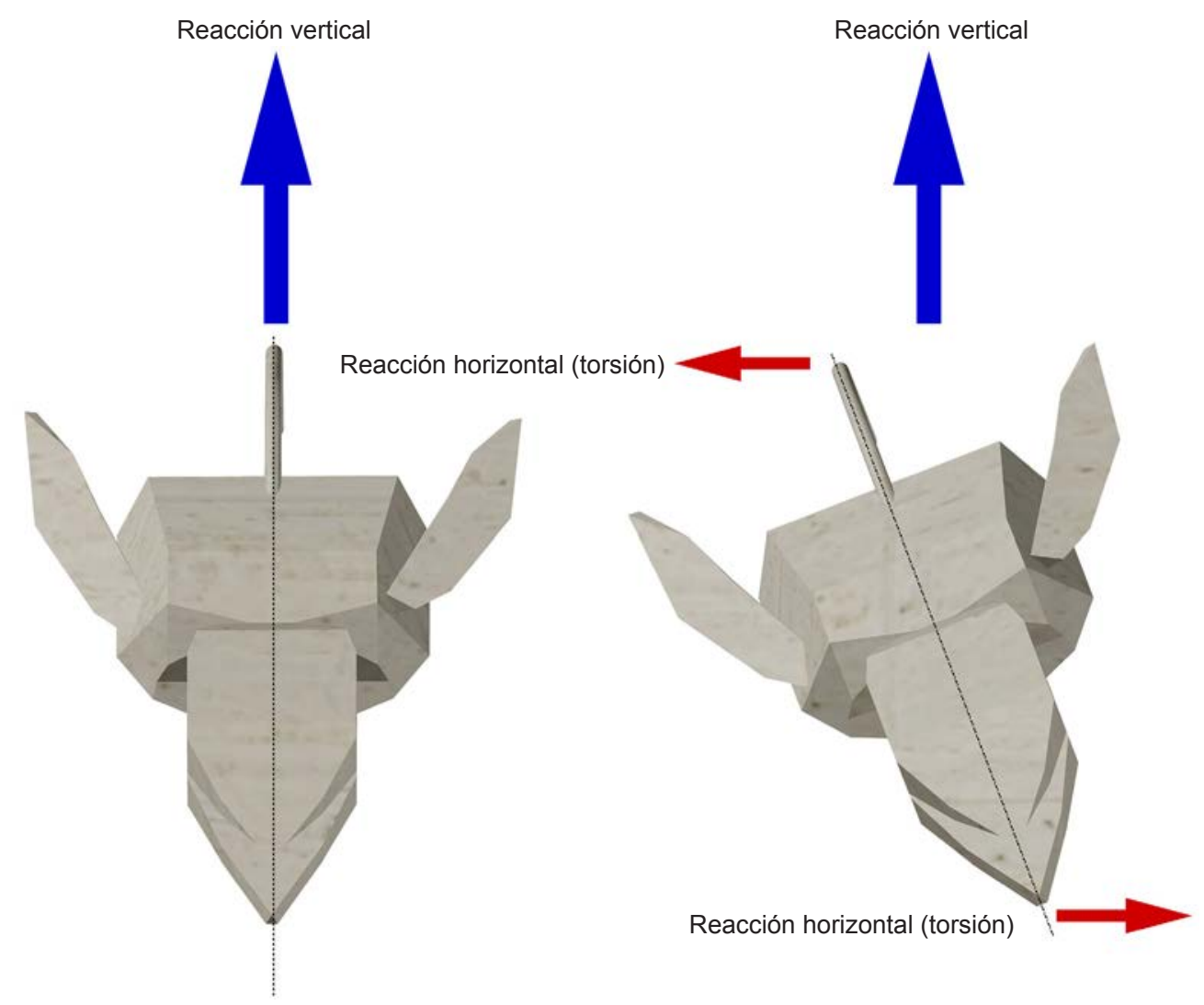

Figura 6. Explicación del fenómeno torsional de la ballena y de las fuerzas necesarias para mantenerla en equilibrio.

ferencia que se consideró fue el de una viga empotrada-articulada. Con esta premisa y considerando siempre que las uniones quedasen integradas en los discos intervertebrales, se procedió a unir en el programa de dibujo cada uno de los cuatro puntos de soporte del techo con cada uno de los nueve puntos de unión resultantes en el tubo, no sobrepasando en ningún caso las longitudes máximas anteriormente descritas. También se dispusieron 6 apoyos en el perímetro de la «Silla». Así se obtienen un total de 52 posibles cables que son necesarios para estabilizar el conjunto. Los cables quedan muy ordenados en el espacio y forman unas superficies regladas que pueden ser entendidas como las generadas en un movimiento de inmersión, aparentando turbulencias. Parecen también haces de luces que iluminan la escena desde cada apoyo, o incluso pueden ser los músculos que le faltan al animal que han sido estirados para mostrar el esqueleto. Recuerda también a un ideograma «Kai Kai» $(24)^{6}$ sujeto por cuatro dedos de un niño. Una vez estaban claras las directrices del predimensionado, se procedió a calcular la estructura.

\subsection{Un problema de cálculo no lineal}

Como los cables llegan muy inclinados al tubo y como las vértebras lo ocultan, es necesario disponer unas barras perpendiculares al tubo en el disco intervertebral, en forma de Y, con tres posibles posiciones: una en cada extremo, y realizar la entrega fuera de la vértebra, evitando interferencias. El primer cálculo se realizó en estático lineal. Puede parecer incorrecto, pero siempre se debe hacer un primer tanteo despreciando los efectos no lineales de los cables, evitando así que el programa llegue a producir inestabilidades: un modelo de cálculo lineal siempre tiene solución, aunque sea inestable, si no es un mecanismo, pero un problema de cálculo no lineal de cables puede no tenerla. Para entender esta última afirmación se puede estudiar el análisis de un cable vertical empotrado en el extremo superior, con una carga que lo intente comprimir.

El cálculo lineal no distingue si una barra trabaja a compresión o a tracción, por lo que si un tirante se comprime no pandea y trabaja a la misma capacidad que si estuviese traccionado. Este fenómeno se produce con frecuencia cuando se coloca una cruz de San Andrés en un pórtico, usando diagonales muy esbeltas: si el cálculo es lineal la deformación final obtenida, que es incorrecta, será aproximadamente la mitad a la deformación que se obtendría si el cálculo fuese no lineal (25).

La propuesta finalmente elegida puede definirse como un caso de hiperestatismo axil puesto que a cada punto del «Arco» le podían corresponder hasta cuatro cables traccionados. El programa de cálculo elegido fue Robot Millennium 2007 (26). Los cables que no se traccionaban se eliminaron del modelo. Este proceso se repitió varias veces, y en cada caso se fueron cambiando de posición de entrega con el tubo, para controlar también la torsión. Cuando la estructura funcionaba tanto a deformación como a tensión, y cuando todos los cables así como el «Arco» quedaban traccionados,

\footnotetext{
${ }^{6}$ Práctica ancestral Rapanui, que consiste en dar forma a un ideograma con hilos que contiene en su imagen una historia, que se narra a través de una recitación denominada «Patautau».
} 


\section{NRd según MEd}

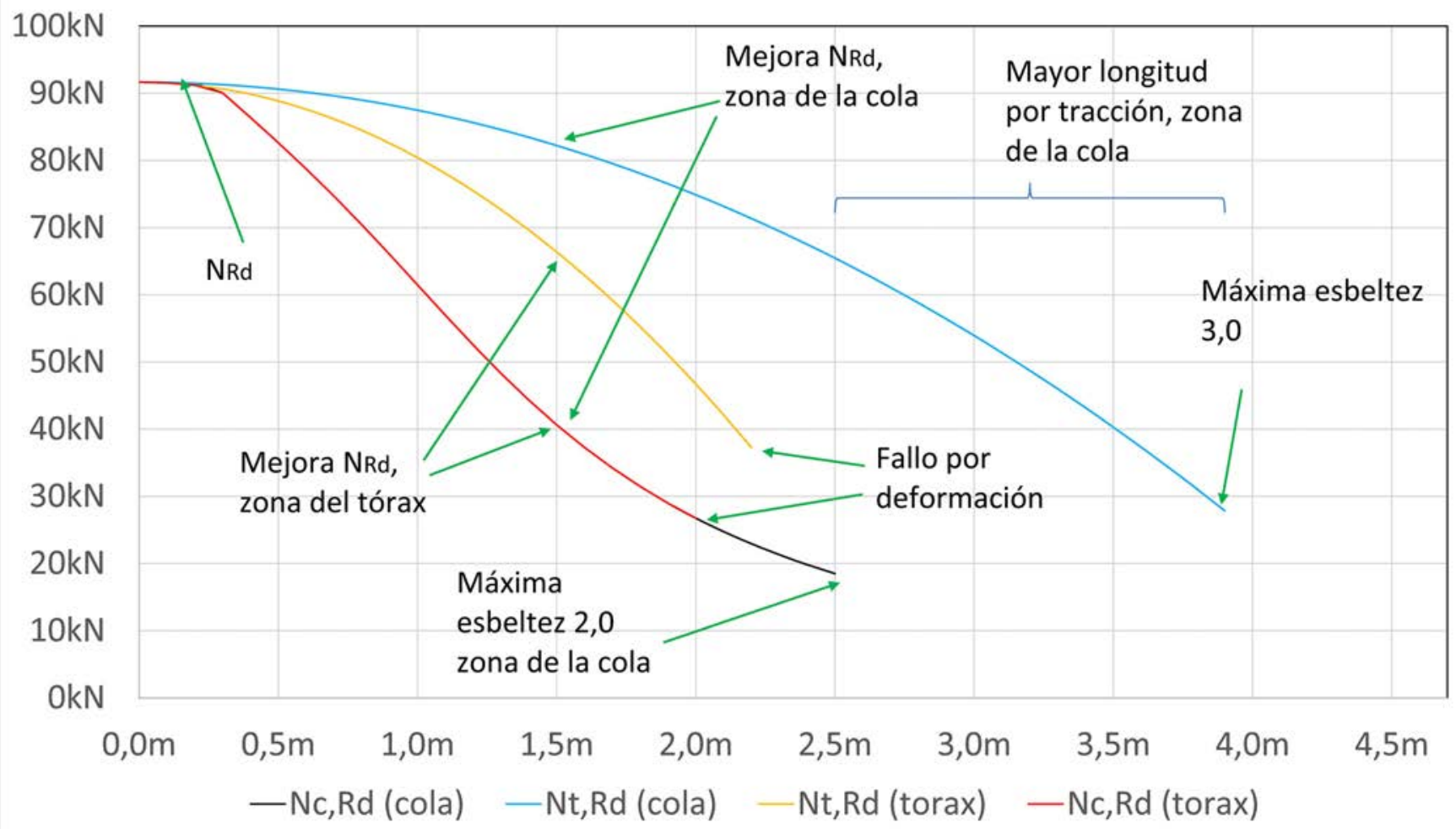

Figura 7. Carga máxima admisible para el tubo en función de su longitud y del Momento Flector.

se pasó a cálculo no lineal de esfuerzos, para comprobar las tracciones impuestas. Algunas de las hipótesis simples no convergían, puesto que en ellas se introducía únicamente el peso de un único hueso importante, sin embargo todas las combinaciones funcionaron correctamente desde el inicio, debiendo variar de posición algún cable, a pesar de haber considerado el cálculo lineal previo. Los resultados de los axiles finales de los cables se pueden analizar en la Figura 8, así como las cuatro reacciones, que en la dirección vertical no llegaban en ninguno de los cuatro apoyos a $8,0 \mathrm{kN}$ en Estado Límite Último.

Al final del proceso de diseño quedaron once cables de $4 \mathrm{~mm}$ de diámetro de acero calidad Y1860S7 en cada apoyo, que además debían ir a eje de nudo de la cubierta. La diferencia de tracciones entre el cálculo lineal y el no lineal fue ligeramente diferente: tan sólo fue necesario corregir la posición de dos cables que dejaban de trabajar.

\subsection{Trabajando el detalle}

El detalle más importante que se debía resolver era el que proponía tres posiciones alrededor del disco intervertebral. Para solucionarlo se diseñó un sistema formado por cuatro varillas roscadas que atravesaban el tubo. Con esta solución se consigue soportar el esqueleto desde dos puntos superiores. Combinando los tres puntos se puede contener el fenómeno torsional que se ha explicado anteriormente. Las tuercas con cáncamo superiores permitían además que en cada extremo se pudiesen colocar varios cables. La solución final se puede ver en la Figura 9.

El detalle de unión con la estructura de cubierta (27) debía de encontrar una solución fácil de ejecutar y que permitiese que los cables tuviesen algo de juego. Si se conseguía encontrar un plano en el que se entregasen todos los cables, se podría colocar una chapa que trabajaría a tracción, longitudinal y transversalmente. No era posible encontrar un plano que contuviese los once cables de cada punto de apoyo, ya que el tórax es una curva definida en un plano más o menos vertical. Aun así, gracias a la conexión mediante grilletes y manguitos tensores, se consiguió que las uniones entre chapa y cable tuviesen capacidad de giro en cualquier dirección, tal como se puede observar en la Figura 10. Así se proyectaron en el espacio cuatro chapas de espesor $10 \mathrm{~mm}$, colocando máximo dos tirantes por agujero y grillete, reduciendo el número de perforaciones a siete por unidad. También se dejó alguna posición más para solucionar imprevistos durante la fase de construcción.

Para unir la mandíbula con la «Silla» y que no deslizase, se colocó una varilla roscada que la atravesaba, pudiendo ser desmontada en un futuro, tal como se aprecia en la Figura 11. La tuerca con cáncamo se colocó para disponer dos de los cables que lo soportan.

Finalmente se añadieron cuatro cables para soportar cada mano, dos en cada una, colgados desde los cables principales. $\mathrm{El}$ peso de estas «manos» es bajo, de aproximadamente 0,4 $\mathrm{kN}$. Buena parte de esta carga queda recogida por la escápula, el hueso que une la mano con el tórax, así que se trata de que la mano no gire contrarrestando en punta tan sólo unos 0,15 $\mathrm{kN}$. Se optó por colocar un cable menor, de $2 \mathrm{~mm}$ de diámetro, sujeto con unos perrillos muy pequeños sobre los cables principales. Así se consigue una unión casi invisible. Los cables secundarios se inclinan en la dirección de los cables principales, consiguiendo que las manos se separasen del tórax, una decisión arquitectónica que favoreció el aspecto de inmersión. 


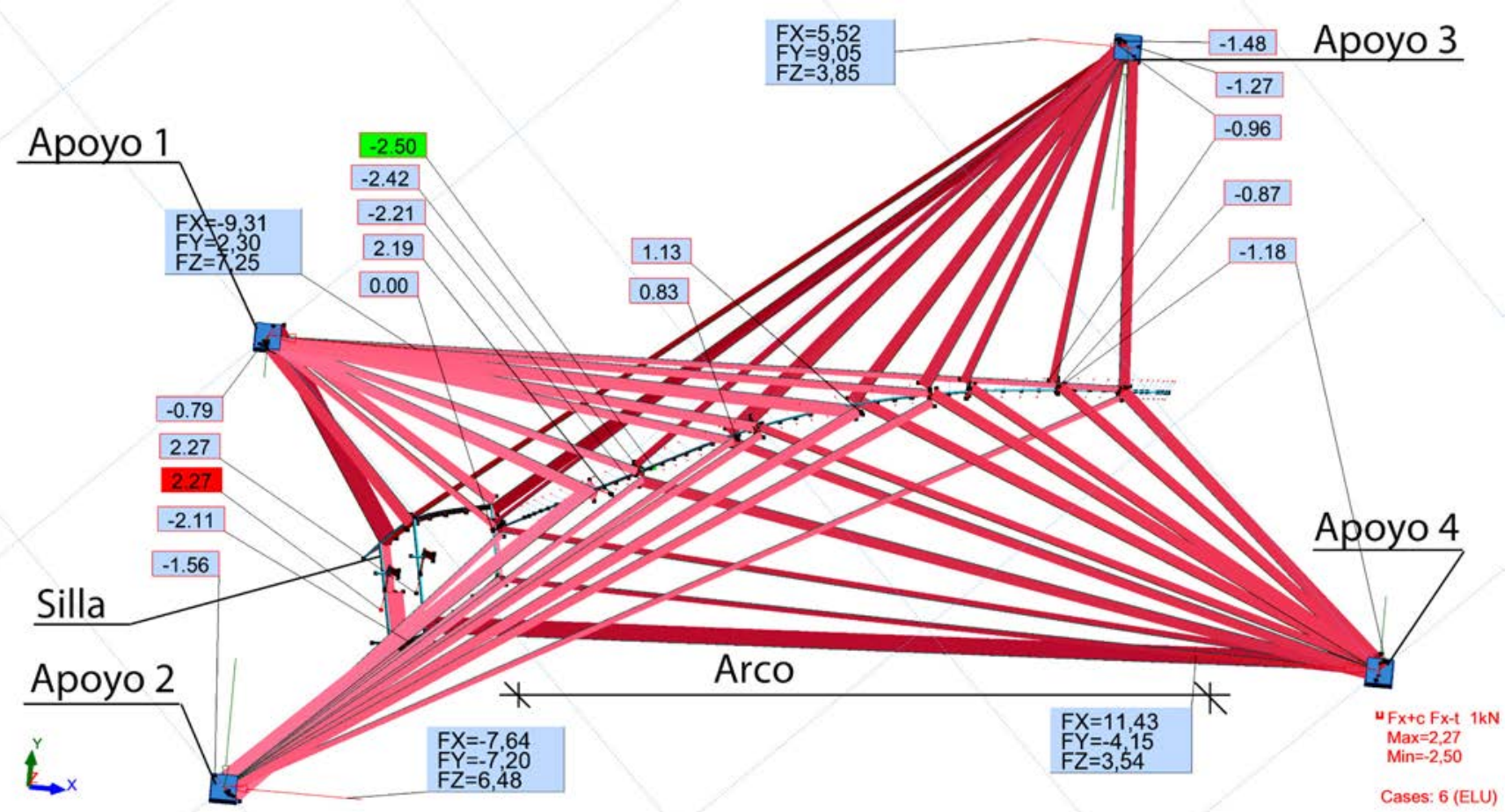

Figura 8. Diagramas de axiles de las barras. El «Arco» siempre queda traccionado para evitar el pandeo. Los esfuerzos de compresión se corresponden con algunas barras transversales que forman parte de las «Y» de unión.

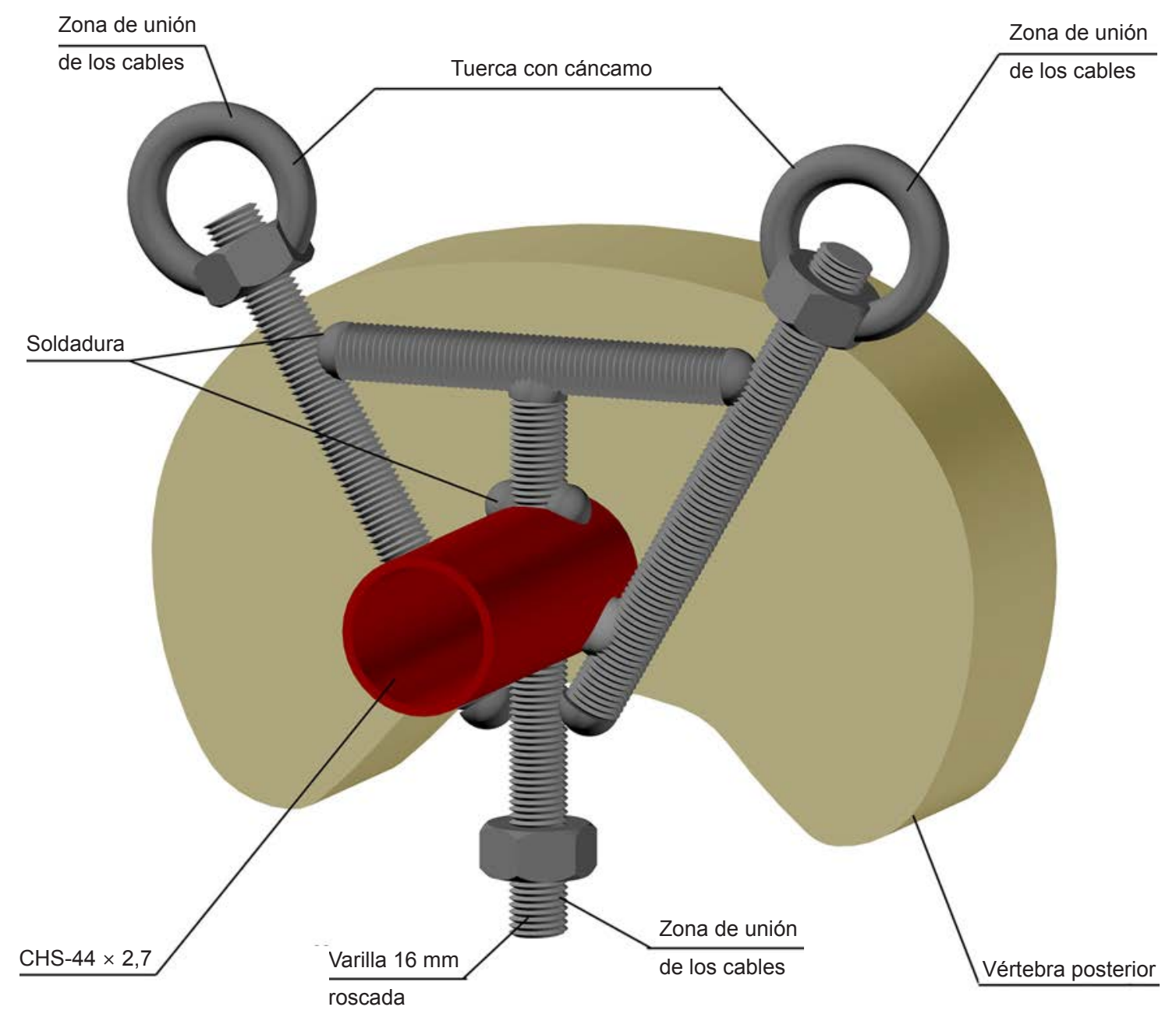

Figura 9. Solución para proveer al tubo de tres sistemas de apoyo en un mismo disco: uno en cada cáncamo, y el tercero en la tuerca inferior. 


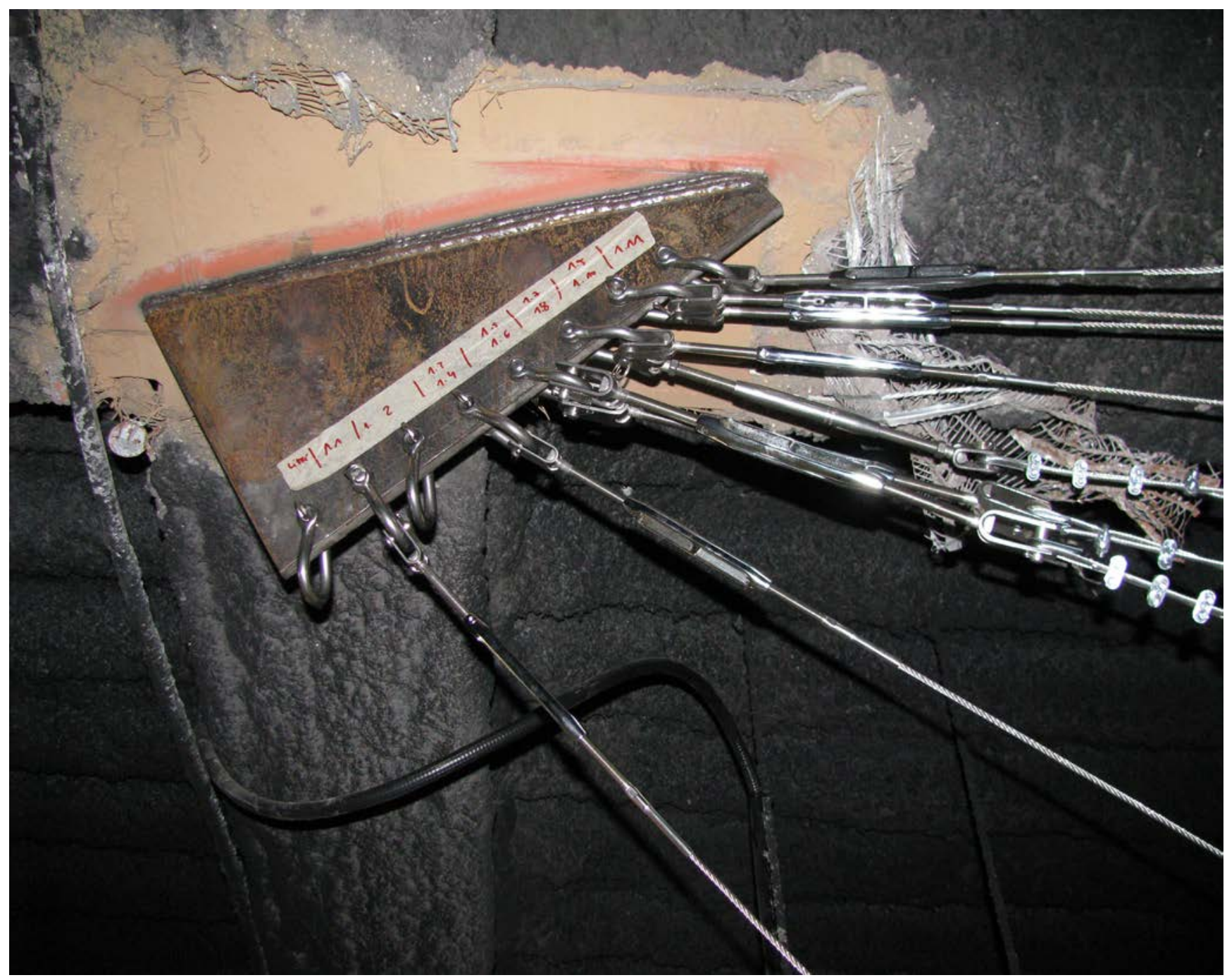

Figura 10. Una de las cuatro chapas para resolver un apoyo, soldada previa eliminación de la vermiculita.

El esqueleto fue «troceado» en cinco partes para facilitar el transporte y su posterior colocación. Para poder acoplarlos entre sí se colocaron unos casquillos tubulares de menor dimensión, haciendo posible el machihembrado entre las piezas. También se dejaron unos agujeros en los extremos del tubo sin mecha, coincidiendo con dos discos intervertebrales consecutivos; una vez las piezas estaban machihembradas, se taladraba el casquillo utilizando por guía los agujeros del tubo para, finalmente, colocar un par de pasadores diámetro $8 \mathrm{~mm}$ en cada unión, que quedarían ocultos por los falsos discos de Porexpán «EPS». Tal como se indica en EN 1993-1-1 (28), estas dos perforaciones hicieron necesario revisar la Figura 7, puesto que la resistencia última de cálculo de la sección transversal neta $\left(\mathrm{N}_{\mathrm{t}, \mathrm{Rd}}\right)$ es un 1,2\% menor a la resistencia plástica de cálculo de la sección bruta $\left(\mathrm{N}_{\mathrm{u}, \mathrm{Rd}}\right)$ según (28), reduciendo la capacidad de la sección únicamente en el caso de tracción. La resistencia a flexión se redujo en las uniones a la capacidad resistente del casquillo tubular interior, puesto que se trata de una sección de menor capacidad a flexión que la exterior. Las resistencias a cortante y a compresión no se vieron afectadas por la presencia de los taladros. Los pasadores soportaban un cortante máximo de $65,5 \mathrm{kN}$ considerando la capacidad de la unión según (29). Este detalle puede acabar de comprenderse en la Figura 12.

\section{PUESTA EN OBRA}

El proceso de montaje se completó en apenas tres días, de viernes tarde a domingo. Para ello se colocó un andamio provisional sobre las escaleras que serviría para poder acceder a cada parte del esqueleto con facilidad. Además se utilizaron un andamio móvil y una plataforma elevadora para poder acceder a los apoyos del techo fácilmente, y una grúa de pequeñas dimensiones para subir las piezas más pesadas del esqueleto, pudiéndolas soportar mientras se iban tensando los cables (30). Las reacciones de esta grúa sobre el suelo fueron revisadas con anterioridad, descartando que el pavimento pudiese romper localmente a punzonamiento, concretamente en las zonas donde las patas se apoyaban únicamente sobre la capa de compresión. Se colocaron chapas de $5 \mathrm{~mm}$ de grosor en las patas para repartir mejor los esfuerzos entre las vigas principales. Las reducidas dimensiones de la máquina hicieron posible subirla a la primera planta por el montacargas del edificio.

Durante el proceso de montaje se fue verificando in situ que las fases no producían esfuerzos importantes con respecto al cálculo inicial, pudiendo introducir ligeras correcciones en el programa de cálculo casi en tiempo real. Primero se montó el tórax para que sirviese de referencia al resto de las piezas. 


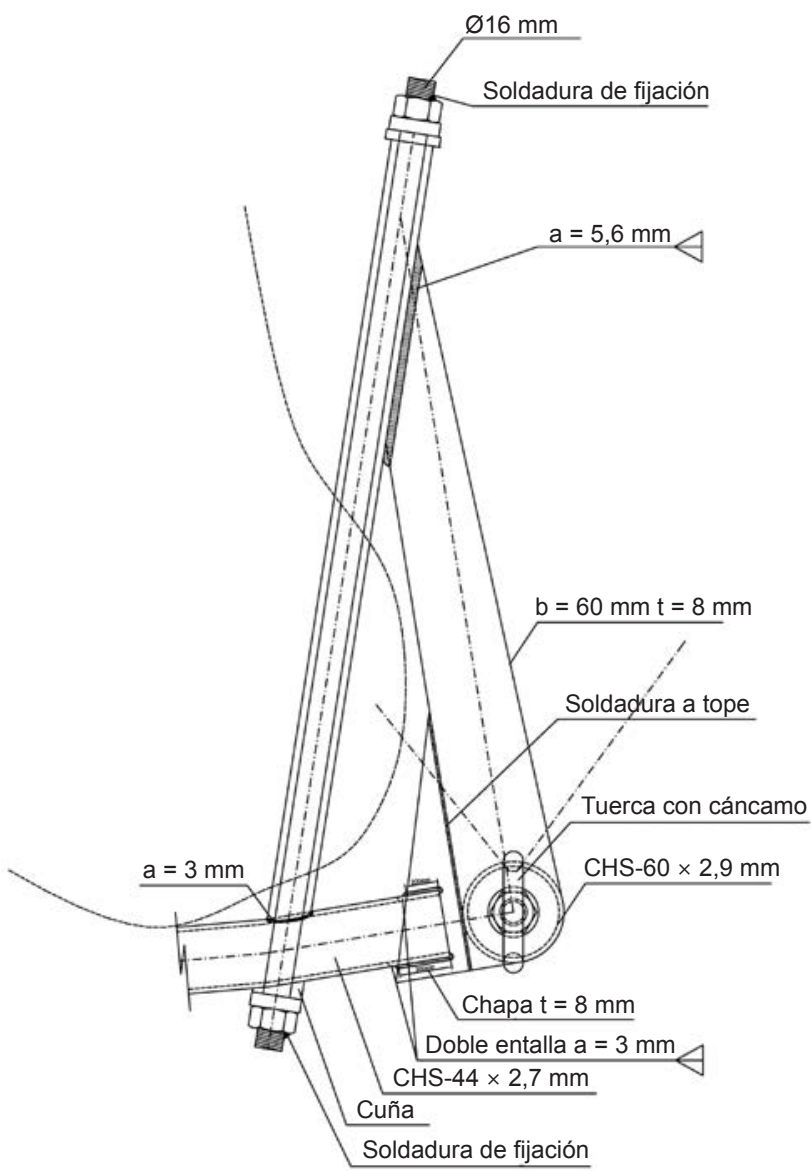

Figura 11. Sistema de fijación de la «Silla» con la mandíbula.
Se comprobó fácilmente que era estable y que estaba en su posición correcta mediante láser, tomando la posición de un caso equivalente en el programa de cálculo. Después se fueron añadiendo las demás piezas que conformaban la cola y no se detectaron movimientos inesperados. Por último se montó la cabeza de una sola vez, tal como se puede apreciar en la Figura 13. Esta pieza, la más pesada, es independiente del resto del cuerpo. Cada vez que se colocaba una pieza se revisaba el par de apriete que se daba a cada cable, para no superar el axil máximo resistente de cada elemento estructural, eliminando así la posibilidad de rotura. El último paso fue montar las «manos» con el sistema que ya se ha explicado, colgándolas de los cables principales.

Todos los cables fueron calculados con un coeficiente de rendimiento mediano para dejar un margen razonable para el posterior tesado manual. No se corrigió la posición tensando los cables para evitar aumentar la tensión de cálculo. En cada extremo de los apoyos se colocaron los manguitos con un sistema de cierre, consiguiendo así que un solo operario pudiese controlar los 11 cables de cada apoyo con una plataforma. El aspecto final del conjunto, antes de retirar el andamio, se puede ver en la Figura 14 donde se puede apreciar que la «Silla» queda integrada en el conjunto (31).

\section{CONCLUSIONES}

En el desarrollo del proyecto se ha establecido una metodología de diseño que puede ser usada en estructuras de características similares a la descrita, concretamente en estructuras ligeras de pequeña envergadura formadas por cables. El método puede constituir un patrón exportable

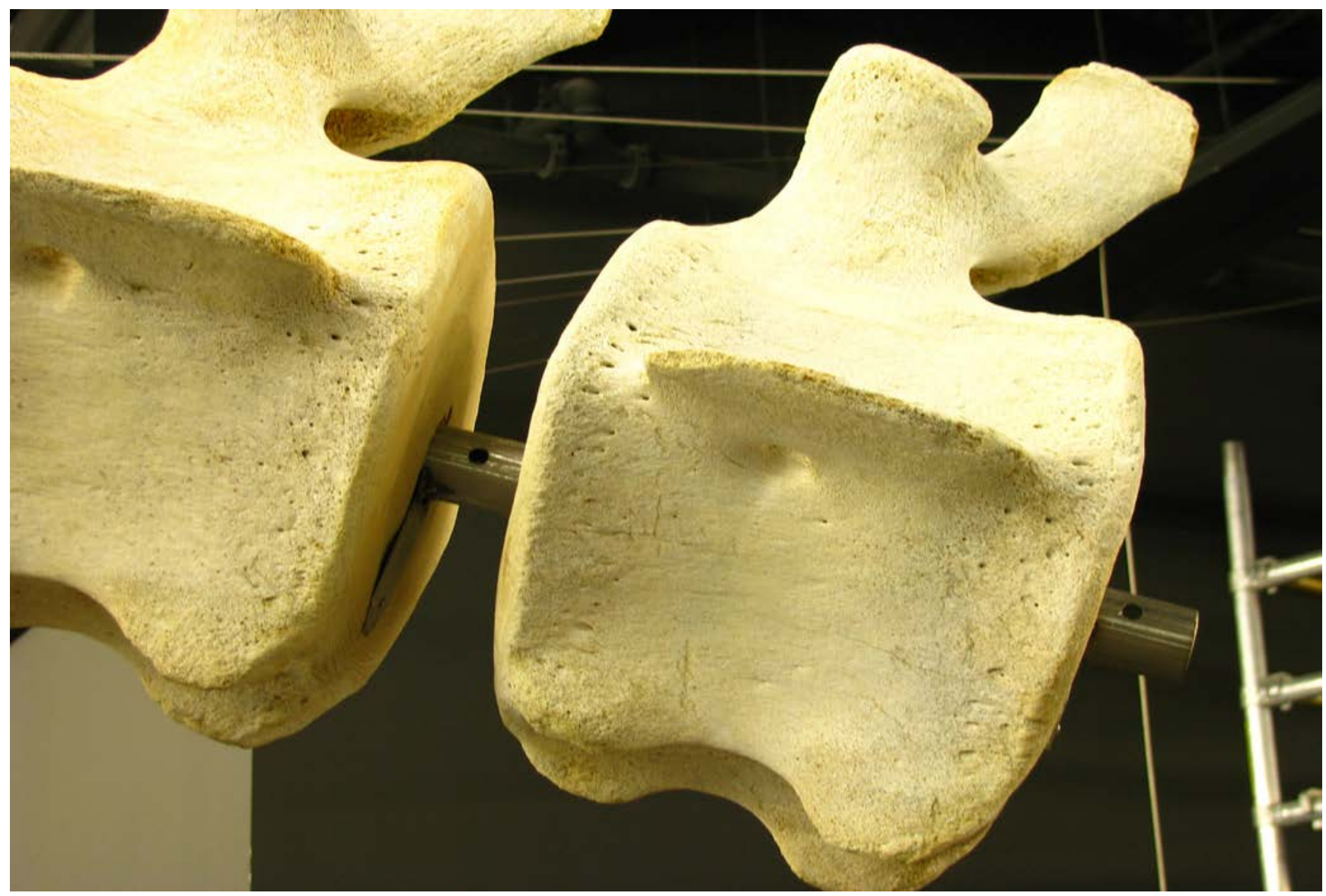

Figura 12. Detalle de las perforaciones que sirven de guía para taladrar el casquillo de la otra pieza, cuando se hace el machihembrado. 


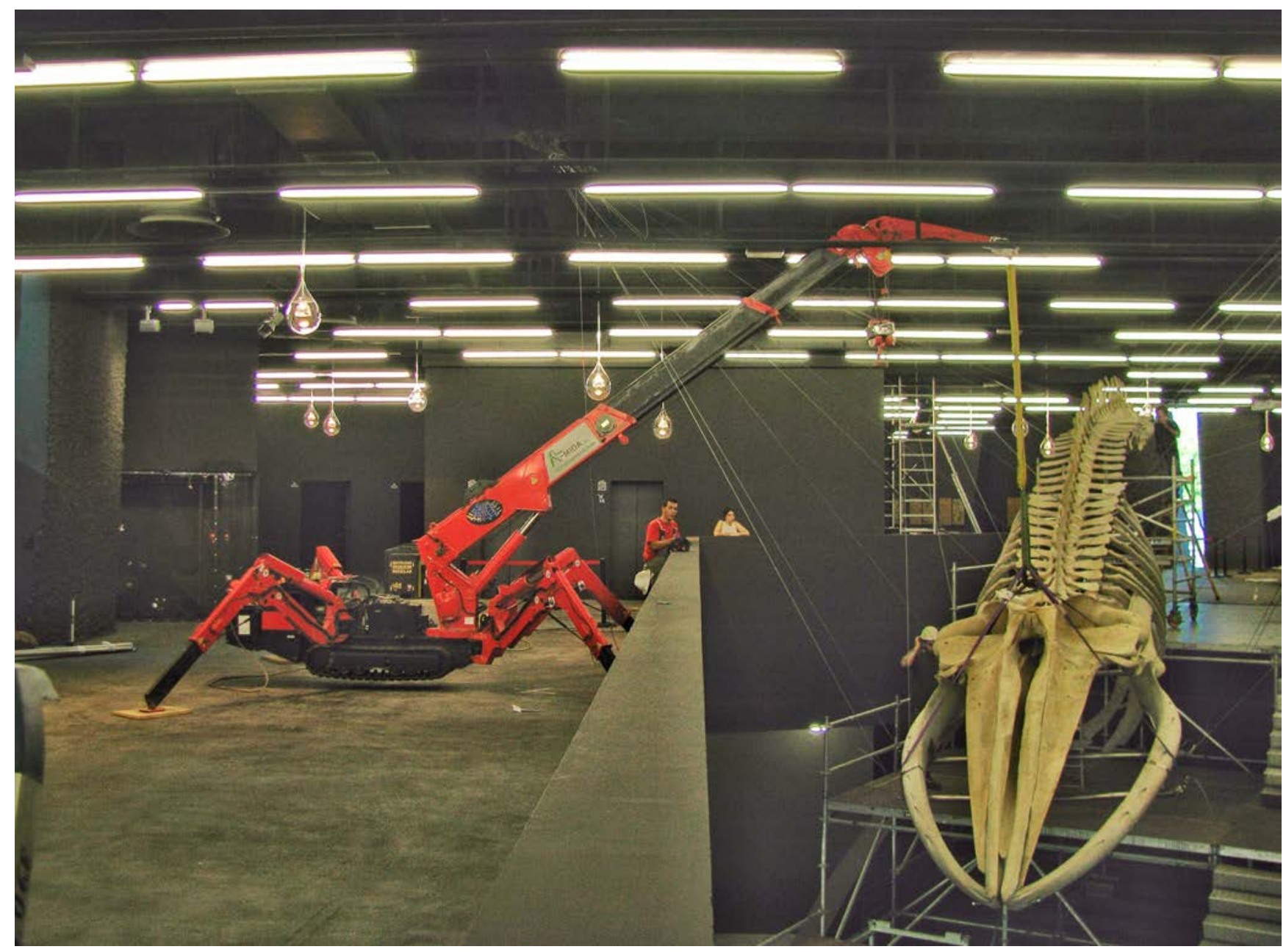

Figura 13. Proceso de montaje de la cabeza, con ayuda de una grúa fija sobre el forjado.

para el análisis de otro tipo de estructuras, pues presenta un camino directo de cómo organizar un proyecto estructural de principio a fin.

Se ha utilizado una estrecha relación entre predimensionado, cálculo lineal y cálculo no lineal para optimizar la solución final, consiguiendo una estructura ligera consecuente a los esfuerzos generados por la decisión de la forma en el espacio. Se ha explicado también una forma razonada de proceder con un programa de cálculo no lineal usando previamente una solución lineal.

Se ha encontrado una manera muy sencilla de dar soportes a los cables gracias a la incorporación de cuatro chapas suficientemente rígidas con una holgura suficiente como para poder dar cabida a hasta 11 cables con los manguitos correspondientes. Esta solución facilitó la rápida puesta en obra.

Así mismo se ha demostrado que en una estructura ligera se pueden obtener soluciones más livianas haciendo trabajar a la estuctural principal a tracción, eliminando no solamente el fenómeno de pandeo sino también reduciendo el pandeo lateral de las piezas flectadas, puesto que las cabezas comprimidas se reducen por efecto de la tracción.

La metodología adoptada, así como las soluciones constructivas elegidas que fueron diseñadas para el proyecto que se

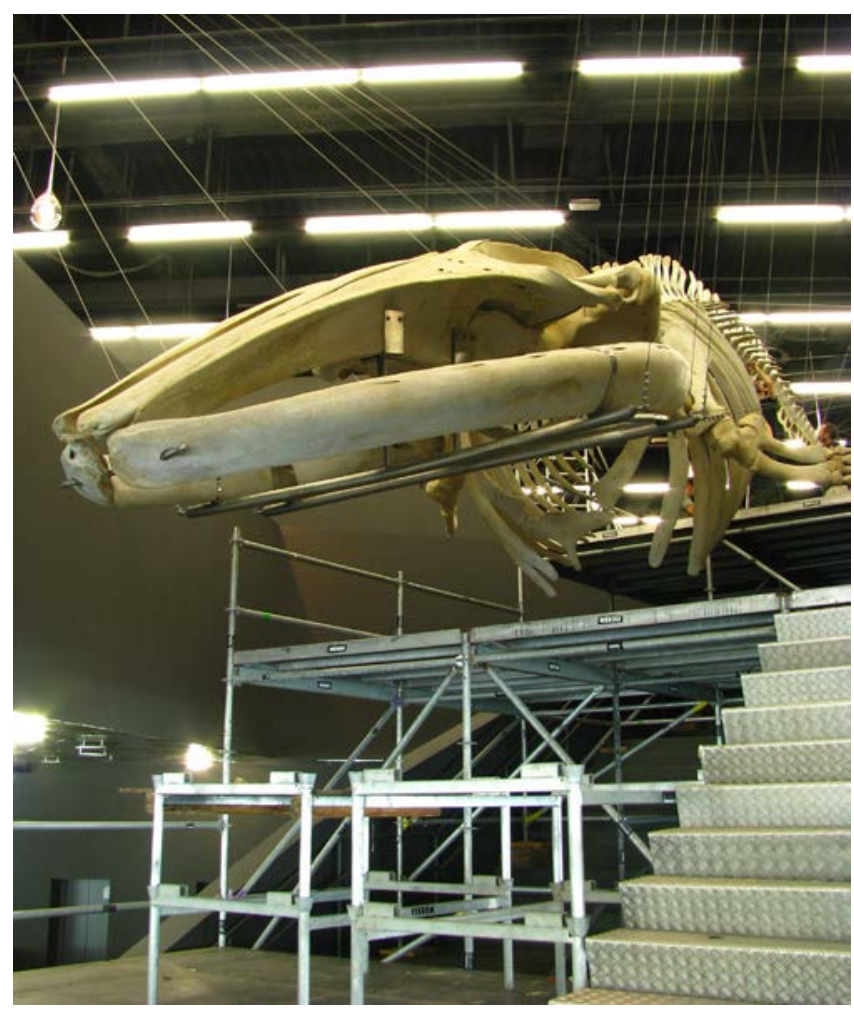

Figura 14. La estructura en carga, antes de ser desmontado el andamio de trabajo. 
describe, permitieron una puesta en obra rápida y eficiente, haciendo posible que el museo cerrase únicamente tres días.

\section{AGRADECIMIENTOS}

Mi agradecimiento personal a la firma de Arquitectos Herzog \& de Meuron. A Miquel del Río Sanín por su continuo apoyo en el proceso de diseño y de montaje de la estructura. A Anna Omedes y a Eulàlia Garcia Franquesa del Museu de Ciències Naturals de Barcelona. A Robert Brufau i Niubó por permitirme colaborar en este proyecto. A Santiago de León Molina, Arquitecto Técnico de la obra, a GROP y a GEA, responsables también del diseño y puesta en obra del proyecto.

\section{REFERENCIAS}

(1) Márquez, F., Levene R. (2006). Herzog \& de Meuron 2002-2006. El Croquis (129/130): 270-283.

(2) Carrillo, M., Alcántara, E., Taverna, A., Paredes, R., García-Franquesa, E. (2014). Descripción osteológica del rorcual común (Balaenoptera physalus, Linnaeus, 1758) del Museo de Ciencias Naturales de Barcelona. Arx Miscel-lània Zoològica, 12: 93-123 [Internet]. Disponible en: http://amz.museucienciesjournals.cat/volum-12-2014-amz/descripcion-osteologica-del-rorcual-comun-balaenoptera-physalus-linnaeus-1758-del-museo-de-ciencias-naturales-de-barcelona/?lang=es.

(3) Le Blond, S., Guilminot, E., Lemoine, G., Huet, N., Mevellec, J. Y. (2009). FT-Raman spectroscopy: A positive means of evaluating the impact of whale bone preservation treatment. Vib Spectrosc., 51(2): 156-161.

(4) Guilminot, E., Lemoine, G., Pelé, C., Poisson, L., Surbled, M., Louvet, I., et al. (2014). Re-treatment of whale bones - How to extract degraded fats from weakened bones? J Cult Herit, 15(2): 128-135 [Internet], Elsevier Masson SAS, doi: http:// dx.doi.org/10.1016/j.culher.2013.03.008.

(5) Rui-Wamba, J. (1998). Aforismos estructurales, 168 pp., Madrid: Fundación Esteyco.

(6) Canarias Conservación, GEA (2011). Estudio osteológico, restauración y montaje del esqueleto MZB 83-3084 [InternetYoutube]. Disponible en: http://www.youtube.com/watch?v=duJof5v1AVU 01/o8/2011.

(7) Yang, H., Jekir, M. G., Davis, M. W., Keaveny, T. M. (2016). Effective Modulus of the Human Intervertebral Disc and its Effect on Vertebral Bone Stress. J Biomech, 49(7): 1-7 [Internet], Elsevier. Disponible en: http://linkinghub.elsevier. com/retrieve/pii/Soo21929016302287.

(8) Rho, J. Y., Kuhn-Spearing, L., Zioupos, P. (1998). Mechanical properties and the hierarchical structure of bone. Med Eng Phys., 20(2): 92-102.

(9) Olszta, M. J., Cheng, X., Jee, S. S., Kumar, R., Kim, Y. Y., Kaufman, M. J., et al. (2007). Bone structure and formation: A new perspective. Mater Sci Eng R: Reports, 58(3-5): 77-116.

(10) Antón, J. (2010). La ballena avista su nueva casa azul. El País digital [Internet]. Disponible en: https://www.google. es/url?sa $=$ t\&rct $=$ j\&q $=\& e s r c=s \&$ source $=$ web\&cd $=1 \& c a d=r j a \& u a c t=8 \& v e d=0 C D A Q F j A A \& u r l=h t t p: / /$ elpais.com $/$ diario/2010/12/18/catalunya/1292638040_850215.html\&ei=wYx1U6S1IoHtogXsnYHgCA\&usg=AFQjCNHipZ2vlz9biVs Ei4PjOvc8QNmbkw\&bvm=bv.

(11) Bletzinger, K.-U., Ramm, E. (2001). Structural Optimization and Form Finding of Lightweight Structures. Comput Struct., 79(22-25): 2053-2062.

(12) Cassinello, P., Schlaich, M., Torroja, J. A. (2010). From thin concrete shells to the 21st century's lightweight structures. Informes de la Construcción, 62(519): 5-26, doi: http://dx.doi.org/10.3989/ic.10.040.

(13) Otto, F., Schanz, S. (1995). Frei Otto, Bodo Rasch : finding form : towards an architecture of the minimal : the werkbund shows Frei Otto, Frei Otto shows Bodo Rasch : exhibition in the Villa Stuck, Munich, on the occasion of the award of the 1992 Deutscher Werkbund Bayern Prize to F [Internet], Stuttgart: Axel Menges [cited 2016 Mar 20], 239 pp. Disponible en: http://cataleg.upc.edu/record=b1122031 S1* cat

(14) Nazarian, A., Müller, R. (2004). Time-lapsed microstructural imaging of bone failure behavior. J Biomech., 37(1): 55-65.

(15) Zwahlen, A., Christen, D., Ruffoni, D., Schneider, P., Schmölz, W., Müller, R. (2015). Inverse Finite Element Modeling for Characterization of Local Elastic Properties in Image-Guided Failure Assessment of Human Trabecular Bone. $J$ Biomech Eng, 137(1): 011012 [Internet]. Disponible en: http://biomechanical.asmedigitalcollection.asme.org/article. aspx?articleID=1930785.

(16) Otto, F. (1967). Tensile structures: design, structure, and calculation of buildings of cables, nets, and membranes, London: MIT Press [Internet] [cited 2016 Mar 20]. Disponible en: http://cataleg.upc.edu/record=b1077503 S1* cat.

(17) Yosida, N. (2006). Herzog \& de Meuron 2002-2006, pp. 270-283, $A+U$.

(18) BSI (2006). BS EN 1993-1-11: Eurocode 3: Design of steel structures. Part 1-11: Design of structures with tension components. British Standards Institution (BSI).

(19) Rocci, S. (1963). Fórmulas simplificadas de dimensionamiento de cables. Informes de la Construcción, $16(153):$ 85-96.

(20) Crespo, P., Bellod, J. L., Rui-Wamba, J. (1995). Aplicaciones del pretensado en las estructuras metálicas. Hormigón y Acero (196): 155-156.

(21) Arroyo Portero, J. C. (2009). Números gordos en el proyecto de estructuras, Madrid: Cinter [Internet] [cited 2016 Mar 20]. Disponible en: http://cataleg.upc.edu/record=b1361728 S1* cat.

(22) Jurado-Piña, R., Salazar-Troya, M. A. (2014). A simple method for the design of tension structures combining topological mapping and nonlinear structural analysis. Informes de la Construcción, 66(EXTRA-1): mo12, doi: http://dx.doi. org/10.3989/ic.13.090.

(23) Costales, I. (2012). El Pretensado en las estructuras de acero. Universitat Politècnica de Catalunya [Internet] [cited 2016 Mar 20]. Disponible en: http://hdl.handle.net/10803/96778. 
(24) Pignet, L. (2001). Kai Kai: Tradition and Innovation on Rapa Nui. En Stevenson, C. M., Lee, G., Morin, F. (Eds.), Proceedings of the 5th International Conference on Easter Island and the Pacific (pp. 373-376). Kamuela, Hawaï, Los Osos (CA): Easter Island Foundation.

(25) Heyman, J., Albuerne, A., Santiago, H. (2011). Teoría básica de estructuras, 150 pp., Madrid: Escuela Técnica Superior de Arquitectura de Madrid [Internet] [cited 2016 Jun 5]. Disponible en: http://cataleg.upc.edu/record=b1389919 S1*cat.

(26) Marsh, K. (2014). Autodesk robot structural analysis professional 2014: essentials: the essential guide to learning autodesk robot structural analysis professional, Somerville, MA: Marsh API [Internet] [cited 2016 Mar 22]. Disponible en: http://cataleg.upc.edu/record=b1463239 S1*cat.

(27) Martin Saiz, R. (2016). Diseño de anillos de compresión no circulares y distribución óptima de fuerzas en el plano. Barcelona: Universitat Politècnica de Catalunya [Internet] [cited 2016 Mar 23]. Disponible en: http://www.tdx.cat/ handle/10803/335273.

(28) AENOR (2010). UNE-EN 1993-1-1:20o8/AC Eurocódigo 3: proyecto de estructuras de acero: Parte 1-1: Reglas generales y reglas para edificios. Madrid: AENOR [Internet] [cited 2016 Mar 20]. Disponible en: http://cataleg.upc.edu/ record=b1378853 $\sim \mathrm{S}^{*}$ cat.

(29) AENOR (2013). UNE-EN 1993-1-8 Eurocódigo 3: Proyecto de estructuras de acero. Parte 1-8: Uniones. Madrid: AENOR.

(30) Gimferrer Vilaplana, X. (2016). Análisis numérico de los efectos del pretesado sobre estructuras textiles laminares tensoestáticas. Barcelona: Universitat Politècnica de Catalunya [Internet] [cited 2016 Mar 23]. Disponible en: http://www. tdx.cat/handle/10803/312845.

(31) Museu Blau NAT (2011). La balena ja ha arribat al Museu Blau. Fotos en Flickr. [Internet Disponible en: https://www. flickr.com/photos/museuciencies/sets/72157627044295321/. 